Bioscientia Medicina: Journal of Biomedicine \& Translational Research

Journal Homepage: www.bioscmed.com

\title{
Cardiac Septal Defect in Children: Hemodynamics, Clinical Manifestations and Detection
}

\author{
Ria Nova ${ }^{*}$, Sukman Tulus Putra ${ }^{2}$, Siti Nurmaini ${ }^{3}$, Radiyati Umi Partan ${ }^{4}$ \\ ${ }^{1}$ Department of Child Health, Division of Pediatric Cardiology, Dr. Moh Hoesin Hospital, Faculty of Medicine, Universitas Sriwijaya, \\ Palembang, Indonesia \\ ${ }^{2}$ Department of Child Health, Division of Pediatric Cardiology, DR. Cipto Mangunkusumo Hospital, Faculty of Medicine, Universitas \\ Indonesia, Jakarta, Indonesia \\ ${ }^{3}$ Intelligent System Research Group, Universitas Sriwijaya, Palembang, Indonesia \\ ${ }^{4}$ Department of Medicine, Dr. Moh Hoesin Hospital, Faculty of Medicine, Universitas Sriwijaya, Palembang, Indonesia
}

\section{A R T I C L E I N F O}

\section{Keywords:}

Cardiac septal defect

Hemodynamics

Clinical manifestations

Detection

\section{*Corresponding author: \\ Ria Nova \\ E-mail address: rialuthfan@yahoo.com}

All authors have reviewed and approved the final version of the manuscript.

\section{https://doi.org/10.32539/bsm.v5i6.393}

\begin{abstract}
A B S T R A C T
Cardiac septal defect in children is one of the congenital heart defects characterized by atrial septal defects (ASD), ventricular septal defects (VSD) and defects in both atrial and ventricular septum (AVSD). The hemodynamic changes that occur are caused by a left to the right shunt. Differences in location, size of the defect and pulmonary vascular resistance make hemodynamic differences and clinical manifestations between the three types of cardiac septal defects. Detection of cardiac septal defects can be done by clinical examination listening to heart sounds and murmurs that are characteristic for each defect. However, clinical examination alone is often still difficult to determine the type of cardiac septal defect so that several supporting examinations such as photo thorax, ECG, echocardiogram and cardiac catheterization need to be done to help establish the diagnosis.
\end{abstract}

\section{Introduction}

Cardiac septal defect is a group of acyanotic congenital heart disease characterized by atrial septal defect (Atrial septal defect $=$ ASD), ventricular septal defect (VSD), as well as defects in both atrial and ventricular septum (Atrioventricular defect $=$ AVSD). ${ }^{1}$ The prevalence of cardiac septal defects varies, VSD is more common than the other two defects, while AVSD is very rare. ${ }^{2}$

Although this abnormality has occurred since the first trimester of intrauterine life, detection after birth is often too late. ${ }^{3}$ One of the causes of delay detection delay is familiar with clinical signs and symptoms arising from the congenital heart disease. ${ }^{3.4}$

Clinical signs and symptoms found in cardiac septal defects vary greatly depending on the size and location of the defect in the cardiac septum. In small defects can be asymptomatic but can still be recognized by listening to the specific heart sounds caused by this cardiac septal defect. ${ }^{1}$ Large defects are often accompanied by easily recognizable shortness of breath or with accompanying poor nutritional status, but if the examiner does not know that this sign is a clue to confirm the presence of a cardiac septal defect, early detection will still go unnoticed. 5 
The following will describe how hemodynamics, clinical manifestations differ between defects in the atrial septum, in the ventricular septum or defects in both septum and their detection.

\section{Hemodynamic}

Cardiac septal defects cause shunts of blood from the left side of the heart to the right side of the heart in the atria or ventricles, known as left to right shunts. ${ }^{6}$ There are two classifications left to right shunt cardiac septal defect atrial shunt at a level that is often referred to as the pre-tricuspid shunt and shunt at the level of so-called ventricular post-tricuspidshunt. ${ }^{7}$ Pretricuspid shunt occurs in ASD whereas post-tricuspid shunt occurs in VSD and AVSD.

The cause of shunts in a pre-tricuspid shunt is different from the shunts that occur in a post-tricuspid shunt. These differences cause hemodynamic differences that occur as well as different clinical manifestations for each cardiac septal defect. ${ }^{6}$ In the pathogenesis of pulmonary vascular damage mechanisms in the pre-tricuspid shunt only determined from the degree and duration of the excessive volume load only. 8 By contrast, the posttricuspid shunt,pulmonary vascular damage mechanisms other than specified by the degree and duration of load volume is also determined by the shear stress on the pulmonary vasculature due to increased blood circulation. ${ }^{8}$

The ASD shunt occurs during the late systolic and early diastolic phases, which increases during atrial contraction and expiration. The magnitude and direction of the shunt is determined by the size of the defect and the relative atrial pressure associated with compliance left and right ventricular. ${ }^{9}$ In a large ASD atrial pressure both relatively the same so that the shunt is dependent ontario compliance between left and right ventricles. ${ }^{9}$ At birth the high pulmonary vascular resistance and compliance is right ventricular low so that the shunt from the left atrium to the right atrium but gradually turned into a reverse condition corresponding age. 8 Shunting ASD causes dilation of the right atrium and right ventricle with increased pulmonary venous return to the left atrium, causing excess volume into the left atrium. Therefore, in ASD there is biatrial volume overload and volume overload to the right ventricle. Long-lasting shunts will cause right atrial reservoir function disorders, myocardial cell hypertrophy, fibrosis and cellular injury. ${ }^{10}$ With the passage of time these hemodynamic changes can lead to the progression of irreversible pulmonary vascular resistance. ${ }^{11}$

The shunt in a VSD is different from the shunt that occurs in ASD. In VSD, the left-to-right shunt across the ventricular septum occurs during the systolic phase (high pressure) so that blood from the left ventricle is physiologically pumped directly into the pulmonary circulation without volume overload in the right ventricle. ${ }^{6}$ The difference is that causes hemodynamic shunt VSD becomes more significant than the shunt on ASD so that pulmonary vascular disease can occur more quickly in the VSD. 6 The amount of shunt flow in a VSD is determined by the size of the defect and the relative resistance of the vascular beds pulmonary and systemic. ${ }^{12}$ In infancy, even with large defects, the number of shunts will be minimal because they are associated with high pulmonary vascular resistance. As pulmonary vascular resistance decreases, the left-to-right shunt across the ventricular septum increases, increasing pulmonary blood flow and causing clinically severe symptoms. ${ }^{13}$

In AVSD hemodynamic changes that occur depending on anatomical abnormalities that accompany, the type of complete generally heavier because shunts occur at the level of atrial septal and ventricular septal so that blood circulation to occur excessive pulmonary and volume overload occurs in both ventricles. ${ }^{14}$ Excessive pulmonary circulation blood flow leads to heart failure and eventually increases pulmonary vascular resistance in a rapid manner. ${ }^{14}$

\section{Clinical manifestations}

In childhood ASD patients are mostly asymptomatic, even cases with large left-to-right shunts do not have obvious symptoms until adulthood. ${ }^{9}$ Tachypnea, difficulty gaining weight, recurrent respiratory infections are rare in infancy. ${ }^{15}$ In 
uncorrected moderate-to-large ASD, the left-to-right shunt of the atrial septum increases with age, leading to volume loading, heart failure, atrial arrhythmias, and/or pulmonary hypertension. ${ }^{15}$

In VSD the clinical manifestations that appear depend on the size of the defect. Small VSDs usually present with an asymptomatic heart murmur. In neonates, a murmur may be detected 4 to 10 days after birth when pulmonary vascular resistance decreases, allowing aoccur left-to-right shunt to. ${ }^{16}$ In moderate to large VSD symptoms of heart failure appear after three to four weeks of life characterized by tachypnea, poor feeding, poor weight gain, tachycardia, hepatomegaly, rhonchi, grunting, retractions and paleness due to peripheral vasoconstriction. 16

In AVSD severity of clinical manifestations and time of onset of symptoms depending on the type of AVSD, interventricular defect size, the resistance ratio between pulmonary and systemic circulation, as well as the severity of the atrioventricular valve regurgitation. ${ }^{1}$ In the incomplete type of AVSD (partial and transitional) most are asymptomatic in childhood. However, symptoms of heart failure will appear in childhood if there is significant mitral valve regurgitation and/or there is excessive pulmonary circulation. ${ }^{1}$ Unlike the case with complete AVSD of both the balanced AV canal type and the unbalanced AV canal type, symptoms appear more rapidly and are more severe. Complete AVSD with channel type balanced AVl symptoms of heart failure have occurred early in life. Clinical symptoms are found in infants from the age of 1 or 2 months which are characterized by tachypnea, poor feeding, poor weight gain, excessive sweating and paleness. If surgery is not performed immediately, pulmonary hypertension will occur immediately as a consequence of excessive pulmonary blood flow. ${ }^{14}$

In complete AVSD unbalanced AV canal type, the amount of blood that passes through the atrioventricular valve is more into one ventricle, causing the other ventricle to become hypoplastic which of course has the potential to reduce blood flow to one of the systemic circulations. ${ }^{17}$ Clinical manifestations that appear depend on the hypoplasia of the ventricles. If the left ventricle is hypoplastic, the symptoms are similar to those of the hypoplastic left heart syndrome (HLHS). ${ }^{14}$ If accompanied by hypoplasia of the aortic valve and the aortic arch will be similar to the symptoms of left-sided lesions obstructed as critical aortic stenosis or aortic coarctation with typical symptoms of low cardiac output (pallor, diaphoresis, dyspnoea, femoral arterial pulsation is weak or non-existent). ${ }^{14}$ These symptoms will manifest when the blood vessel ductus arteriosus closes. When the right ventricle is hypoplastic, the prominent clinical symptoms are cyanosis due to reduced blood flow to the pulmonary circulation and right-to-left shunt.

\section{Detection}

Detection of cardiac septal defects in children is currently still done manually by physical examination listening to heart sounds and murmurs as well as carrying out several supporting examinations such as chest X-ray, ECG, echocardiogram, and cardiac catheterization. ${ }^{18}$ However, diagnostic catheterization examinations are rarely performed because they are invasive, so they are only performed in certain doubtful cases.

Each cardiac septal defect on physical examination gives different characteristic signs. Cardiac auscultation as a reliable method of differentiating cardiac septal defect murmurs should be carried out systematically by evaluating the heart rate and rhythm. ${ }^{19}$ The identification of murmurs in cardiac septal defects is very important and requires skill at the time of clinical examination. The murmur of a cardiac septal defect is often the first indication of this disorder. Physical examination of ASD was characterized by normal heart sound 1 , heart sound 2 heard wide fixed split, and a systolic murmur above the pulmonary valve. ${ }^{1}$ In an ASD with a large defect, a diastolic murmur at the left lower sternal edge may also be heard due to blood flow over the tricuspid valve. ${ }^{1}$ In contrast to VSD, physical examination revealed normal 1 and 2 heart sounds and a holosystolic murmur at the lower left sternal edge. ${ }^{12}$ If the defect is large, a diastolic murmur will be heard over the mitral valve. ${ }^{12}$ In AVSD 
the audible heart sounds and murmurs are almost the same as those found in VSDs, but different if the AVSD has a large VSD component and the pressures of both ventricles are the same, the murmur will not be heard. ${ }^{1}$

Although not specific and the picture varies depending on the size of the defect, chest X-ray and ECG can be used to help detect cardiac septal defects. Although at this time for a definite diagnosis of cardiac septal defect can be done echocardiography examination, but examination of the chest X-ray and or ECG is still an option in the initial evaluation of heart murmurs. ${ }^{21}$ Both show characteristic findings in cardiac septal defects, especially in moderate to large defects. Chest X-ray of cardiac septal defect will show cardiomegaly with increased pulmonary vascular markings. ${ }^{21}$ However, in the case of cardiac septal defects who had suffered irreversible pulmonary hypertension cardiomegaly will disappear, corakan decreased pulmonary vascular and pulmonary segments stand out.11

The characteristic ECG of ASD is characterized by a high $\mathrm{P}$ wave indicating right atrial enlargement, incomplete right bundle branch block (IRBB), and right axis deviation. ${ }^{22}$ However, in contrast to the primum type ASD, the cardiac axis will deviate to the left and superior. ${ }^{22}$ The rhythm is usually sinusoidal, but in adults it may be atrial flutter or fibrillation. If ASD is accompanied by pulmonary hypertension, it will show right ventricular hypertrophy. In VSD the ECG picture is different from ASD and AVSD. In VSDs with moderate to large defects, the ECG shows left ventricular hypertrophy characterized by high $R$ waves in V5 and V6 or in conductions II, III and aVF. ${ }^{16}$ In AVSD marked characteristic ECG heart axis to the left and almost the same superior primum ASD type, but in AVSD obtained hypertrophy of the right atrium and left ventricle hypertrophy arising due to atrioventricular valve regurgitation significance. 23

Echocardiogram is the most important examination and should be done to determine the type of cardiac septal defect. ${ }^{24}$ Even because of the high incidence of AVSD in infants with Down syndrome, all infants with Down syndrome even if there is no heart murmur or any signs and symptoms should undergo an echocardiogram to confirm this abnormality. ${ }^{23}$ Multiple views of the echocardiogram should be performed to assess abnormalities in the cardiac septal defect. In ASD, the atrial septum should be assessed on a subcostal, apical and parasternal view to determine the size of the defect, the type of defect and the relationship of the defect to surrounding tissues. ${ }^{24,25}$ In VSD, the ventricular septum should be assessed on a parasternal short axis and apical four chamber view so that the size of the defect, the type of defect and the relationship with the aortic and pulmonary vessels can be determined.24.26 In AVSD, assessment of the atrial and ventricular septum should be performed on a subcostal, apical, parasternal long axis and parasternal short axis view so that the size of the defect, type of defect, relationship with atrioventricular valves and blood vessels can be known. ${ }^{24}$

Currently, many advanced technologies for computer-based diagnostic systems in cardiology have been developed using artificial intelligence (AI). ${ }^{27}$ Through this technology, an echocardiogram examination can be performed automatically so that it can be used to assist doctors in early detection before referring to a cardiologist for further treatment. The results of an AI-based echocardiogram examination will be more uniform and objective in determining structural and functional abnormalities of the heart so that diagnosis is more accurate. ${ }^{28}$ However, for cardiac septal defects this advanced technology still needs to be developed and is still in the research stage. In the future, the findings of this technology will certainly be very helpful in detecting congenital heart disease and will change the detection method that was previously done manually to switch to an automatic detection method which will simplify and greatly assist doctors in diagnosing congenital heart disease.

\section{Conclusion}

Cardiac septal defect is one of the cyanotic congenital heart defects characterized by defects in the atrial septum, ventricular septum or both septum. Differences in the location and size of the septal defect and pulmonary vascular resistance make hemodynamic changes, clinical manifestations and 
methods of detection of each cardiac septal defect different. To confirm the diagnosis of each type of cardiac septal defect and to determine the accompanying complications, an echocardiogram examination is the standard that must be done.

\section{References}

1. Puri K, Allen HD, Qureshi AM. Congenital Heart Disease. PediatrRev.2017;38(10):471486. doi:10.1542/pir.2017-0032

2. Liu Y, Chen S, Zu L, et al. Global birth prevalence of congenital heart defects 1970 2017 : updated systematic review and metaanalysis of 260 studies. Int $J$ Epidemiol. 2019;48(2):455-463. doi:10.1093/ije/dyz009

3. Rashid U, Qureshi AU, Hyder SN, Sadiq M. Pattern of congenital heart disease in a developing country tertiary care center : Factors associated with delayed diagnosis. Ann Ped Cardiol. 2016;9:210-215. doi:104103/0974-2069.189125

4. Murni IK, Wirawan MT, Patmasari L, Sativa ER, Arafuri N. Delayed diagnosis in children with congenital heart disease : a mixed-method study. BMC Pediatrics. 2021;21:1-7.

5. Iyer PU, Moreno GE, Fernando Caneo L, Faiz T, Shekerdemian LS, Iyer KS. Management of late presentation of congenital heart disease. Cardiol Young. 2017;27(S6):S31-S39. doi:10.1017/S1047951117002591

6. Upadhyay.J, Tiwari N, Rana M, Rana A, Durgapal S BS. Pathophysiology, Etiology, and Recent Advancements in the Treatment of Congenital Heart Disease. J Indian coll cardiol. 2019;9:67-77. doi:10.4103/JICC.JICC

7. Vijayalakshmi I. Evaluation of Left to Right Shunts by the Pediatrician : How to Follow, When to Refer for Intervention ? Indian $J$ Pediatrics. Published online 2015. doi:10.1007/s12098-015-1861-9

8. Kung GC TJ. Pathophysiology of left-to-right shunts. UptoDate. Published online 2020:1-16. Accessed February 16, 2020. https://www.uptodate.com/contents/pathop hysiology-of-left-to-right-

shunts $/$ print? sectionName=Pulmonary

hypertension\&search=pulmonary vascular

obstructive disease in atrial septal defect\&topicRef=105507\&anchor=H8\&source

= see $\_$link

9. Alkashkari W, Albugami S, Hijazi ZM. Current practice in atrial septal defect occlusion in children and adults. Expert Rev Cardiovasc Ther. 2020;18(6):315-329. doi:10.1080/14779072.2020.1767595

10. Gloan L Le, Legendre A, Iserin L, Ladouceur M. Pathophysiology and natural history of atrial septal defect. J Thorac Dis. 2018;10(24):S285463. doi: $10.21037 /$ jtd.2018.02.80

11. Pascall E, Tulloh R. Pulmonary hypertension in congenital heart disease. Future Cardiol. 2018;14(4):343-353.

12. Spicer DE, Hsu HH, Covu J, Anderson RH, Fricker FJ. Ventricular septal defect. OJRD. 2014;9:1-16. doi:10.1186/s13023-014-01442

13. Jortveit J, Leirgul E, Eskedal L, et al. Mortality and complications in 3495 children with isolated ventricular septal defects. Arch Dis Child. 2016;101(9):808-813. doi:10.1136/archdischild-2015-310154

14. Craig E Fleishman M, Aykut Tugertimur M. Clinical manifestations, pathophysiology, and diagnosis of atrioventricular (AV) canal defects. Update. 2020;21:1-38.

15. Vick AGW, Bezold LI. Isolated atrial septal defects (ASDs) in children : Classification, clinical features, and diagnosis. UptoDate. Published online 2020:1-27.

16. Fulton ADR, Saleeb S. Isolated ventricular septal defects in infants and children: Anatomy, clinical features, and diagnosis. UptoDate. Published online 2020:1-26.

17. Overman DM, Baffa JM, Cohen MS, et al. Unbalanced Atrioventricular Septal Defect: Definition and Decision Making. World $J$ Pediatric Congenit Hear Surg. 2010;1(1):91-96. doi:10.1177/2150135110363024 
18. Sun R, Liu M, Lu L. Congenital Heart Disease:

Causes, Diagnosis, Symptoms, and Treatments. Cell Biochem Biophysics. 2015;72(3):857-860. doi:10.1007/s12013015-0551-6

19. Begic Z, Dinarevic SM, Pesto S, Begic E, Dobraca A, Masic I. Evaluation of diagnostic methods in the differentiation of heart murmurs in children. Acta Inf Med. 2016;24(2):94-98. doi:10.5455/aim.2016.24.94-98

20. Kostopoulou E, Dimitriou G, Karatza A. Cardiac Murmurs in Children: A Challenge For The Primary Care Physician. Curr RevPediatr.2019;15(3):131-138. doi:10.2174/1573396315666190321105536

21. Danford DA, Gumbiner $\mathrm{CH}$, Martin AB, Fletcher SE. Effects of Electrocardiography and Chest Radiography on the Accuracy of Preliminary Diagnosis of Common Congenital Cardiac Defects. Pediatric Cardiol. 2000;21:334-340. doi:10.1007/s002460010075

22. Bradley EA, Zaidi AN. Atrial Septal Defect. Cardiol Clin. 2020;38(3):317-324. doi:10.1016/j.ccl.2020.04.001

23. Fleishman C TA. Clinical manifestations, pathophysiology, and diagnosis of atrioventricular (AV) canal defects. UpToDate. Published online 2019:1-38.

24. Deri A, English K. EDUCATIONAL SERIES IN CONGENITAL HEART DISEASE: Echocardiographic assessment of left to right shunts: atrial septal defect, ventricular septal defect, atrioventricular septal defect, patent arterial duct. Echo Res Pr. 2018;5(1):R1-R16. doi:10.1530/erp-17-0062

25. Silvestry FE, Cohen MS, Armsby LB, et al. Guidelines for the Echocardiographic Assessment of Atrial Septal Defect and Patent Foramen Ovale: From the American Society of Echocardiography and Society for Cardiac Angiography and Interventions. $J$ Am Soc Echocardiogr. 2015;28(8):910-958. doi:10.1016/j.echo.2015.05.015

26. Kavanaugh-mchugh AA. Echocardiographic evaluation of ventricular septal defects. UpToDate. Published online 2019:1-31.

27. Gandhi S, Mosleh W, Shen J, Chow CM. Automation, machine learning, and artificial intelligence in echocardiography: A brave new world. Echocardiography. 2018;35(9):14021418. doi:10.1111/echo. 14086

28. Dey D, Slomka PJ, Leeson P, et al. Artificial Intelligence in Cardiovascular Imaging. $\mathrm{J} \mathrm{Am}$ Coll Cardiol. 2019;73(11):1317-1335. doi:10.1016/j.jacc. 2018.12.054 